\title{
RIESGO SOCIAL COMO FACTOR DE RIESGO PARA LA NO ADHERENCIA FARMACOLÓGICA EN PACIENTES CON DM2 MAYORES DE 65 AÑOS
}

\author{
Alfredo Lara-Morales \\ Profesor de Tiempo Completo del Departamento de Enfermería y Obstetricia \\ Universidad de Guanajuato, Guanajuato, México \\ alfred9087@gmail.com \\ Lucia Caudillo-Ortega \\ Profesor de Tiempo Completo del Departamento de Enfermería y Obstetricia \\ Universidad de Guanajuato, Guanajuato, México \\ Ma. Teresa Pratz-Andrade \\ Profesor de Tiempo Completo del Departamento de Enfermería y Obstetricia \\ Universidad de Guanajuato, Guanajuato, México \\ María Aurora Montañez-Frausto \\ Profesor de Tiempo Completo del Departamento de Enfermería y Obstetricia \\ Universidad de Guanajuato, Guanajuato, México \\ Ma. Elvira Moreno-Pulido \\ Profesor de Tiempo Completo del Departamento de Enfermería y Obstetricia \\ Universidad de Guanajuato, Guanajuato, México \\ Víctor Manuel Ramos-Frausto \\ Profesor de Tiempo Completo del Departamento de Enfermería y Obstetricia \\ Universidad de Guanajuato, Guanajuato, México.
}

Recepción Artículo: 15 noviembre 2020 Admisión Evaluación: 23 noviembre 2020 Informe Evaluador 1: 27 noviembre 2020 Informe Evaluador 2: 29 noviembre 2020 Aprobación Publicación: 27 noviembre 2020

\section{RESUMEN}

Objetivos: Establecer la influencia del riesgo social en la adherencia terapéutica al tratamiento farmacológico de la diabetes mellitus 2 (DM2) en mayores de 65 años. Método: Estudio descriptivo transversal a partir de las historias clínicas electrónicas de Atención Primaria en personas mayores de 65 años con DM2 de la Dirección

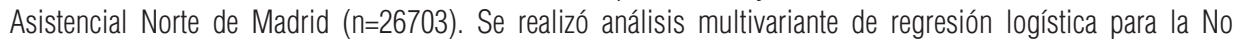
Adherencia al Tratamiento (No AdhT) y para mal control de DM2, desagregado por sexo. Resultados: Un 33,7\% tenían mal control de la DM2 y un 3,1\% No AdhT. En modelos de regresión para el mal control de la DM2, I0 aumentaba la No AdhT (OR: 1,64; IC95\%: 1,07-2,51)). En ambos sexos, el riesgo social provoca una mayor falta de adherencia, en mujeres ( $5.7 \%$ vs. $1.9 \%, p=0.000)$, hombres (7.5\% vs. $2.3 \%, p=0,000)$. En los modelos de regresión para la no AdhT, la aumentaba tener riesgo social (OR: 2.92, IC 95\%: 1.61-5.30) en mujeres; riesgo social ( $O R=3.52$, IC 95\% 1.90-6.52) se asoció con la no adherencia también en hombres. Conclusiones: En los mayores de 65 años con DM2, aunque el No AdhT es bajo, la prevalencia de control deficiente es alta. Para la no AdhT, el riesgo social se presenta como factor de riesgo para ambos sexos, pero tener depresión aumenta la no 


\section{RIESGO SOCIAL COMO FACTOR DE RIESGO PARA LA NO ADHERENCIA FARMACOLÓGICA EN PACIENTES CON DM2 MAYORES DE 65 AÑOS}

AdhT en las mujeres y los síntomas de ansiedad en los hombres. Sin AdhT aumenta el riesgo de un control deficiente de DM2, especialmente en mujeres; además de no hacer dieta y tener sobrepeso/obesidad.

Palabras clave: diabetes mellitus tipo 2; riesgo social; adherencia terapéutica; depresión

\section{ABSTRACT}

Social risk as a risk factor for medication non-adherence in patients over 65 years old with type 2 diabetes mellitus. Aims: The aim of this study was to establish the influence of social risk and its relation on therapeutic adherence to pharmacological treatment of diabetes mellitus type 2 (DM2) in people older than 65 years. Method: A cross-sectional descriptive study based on the electronic clinical records of primary care in people over 65 years of age with DM2 of north area of Madrid $(n=26703)$. Multivariate logistic regression analysis was performed for Non-Adherence to Treatment (Non-AdhT) and for poor control of DM2, disaggregated by sex. Results: A 33,7\% had poor control of DM2 and 3.1\% Non-AdhT. In regression models for poor DM2 control, the Non-AdhT increased (OR: 1.64; 95\% Cl: 1.07-2.51). In both sexes, social risk causes a greater lack of adherence, in women ( $5.7 \%$ vs. $1.9 \%, p=0.000)$, men (7.5\% vs. $2.3 \%, p=0.000)$. In the regression models for non-AdhT, it was increased to have a social risk (OR: 2.92, 95\% Cl: 1.61-5.30) in women; social risk (OR = 3.52, 95\% Cl 1.90-6.52) was associated with non-adherence also in men. Discussion: In those older than 65 years with DM2, although the No AdhT is low, the prevalence of poor control is high. For Non-AdhT, social risk is a risk factor for both sexes, but having depression increases Non-AdhT in women, and anxiety symptoms in men. No AdhT raises the risk of poor control of DM2, especially in women; as well as not dieting and being overweight/obese.

Keywords: type 2 diabetes mellitus; social risk; treatment adherence and compliance; depression.

\section{INTRODUCCIÓN}

Según la OMS, en 2017 había más de 451 millones de personas de entre 18-99 años con diabetes mellitus (DM) en el mundo (Cho et al., 2018). La prevalencia mundial de la enfermedad en personas mayores de 18 años ha aumentado del 4,7\% en 1980 al 8,5\% en 2014, más del 80\% de las muertes por diabetes se registran en países de ingresos bajos y medios; se prevé que las muertes por DM se dupliquen para 2030, y casi la mitad de esas muertes corresponden a personas de menos de 70 años, y un 55\% a mujeres (WHO, 2018)(WHO, 2016)(Risk \& Collaboration, 2016).

En España, la DM es un problema de salud de alta prevalencia en personas mayores de 65 años (a nivel nacional (Basterra-Gortari FJ, 2017): 21,3\% de 65 a 74 años, 25\% en hombres y 18\% en mujeres; en Madrid (Gandarillas Ana María, Valero Virginia, 2018): 25,2\% de 60 a 69 años, 32,8\% en hombres y 18,3\% en mujeres). En lo que respecta a la mortalidad, alrededor de 10000 personas mayores de 65 años mueren anualmente por esta causa en Españas (Joseph \& Golden, 2018); en 2016, en Madrid se produjeron 367 muertes por DM en mayores de 65 años 2016 (Joshep JJ, 2017).

La diabetes es un problema crónico que se caracteriza por la comorbilidad con múltiples enfermedades como las relacionadas con la salud mental (Joshep JJ, 2017). Los riesgos psicosociales que se van acumulando a lo largo de toda la vida, en especial en procesos crónicos como la DM2, aumentan las posibilidades de padecer una pobre salud mental y una muerte prematura; la DM aumenta el riesgo de presentar depresión (Rotella F, 2013) así como de presentar deterioro en las relaciones sociales, lo que sucede de una manera bidireccional, porque no se conoce con certeza sí la enfermedad provoca las malas relaciones o si la falta de apoyo social es el que desencadena enfermedades como la diabetes (Garcia, Castañeda, Allison, Elder, \& Talavera, 2019).

El estudio DAWN (diabetes attitudes Wishes And Needs), encontró asociación de la DM como causa de múltiples problemas psicosociales que interfieren en el autocuidado (control glucémico) (Funnell, 2006). Es común que la no adherencia al tratamiento farmacológico sea elevada en pacientes con DM y depresión (Axon, 2016) así como aquellos que carecen de apoyo social. Por otro lado, intervenir para reforzar la autoeficacia y el apoyo social puede disminuir el efecto negativo de la depresión en la adherencia farmacológica según estudios realizados(Tovar, Rayens, Gokun, \& Clark, 2013)(Osborn, Ph, H, Egede, \& S, 2012).Así mismo, la literatura indica que 
el afrontamiento de factores de riesgo como los problemas psicosociales, incluyendo la falta de apoyo social, conducen a resultados positivos en la diabetes, como por ejemplo una mejor adherencia farmacológica (Smalls et al., 2012)(Koetsenruijter et al., 2016).

El apoyo social aparece como un elemento indispensable cuando hablamos de enfermedades crónicas como la DM2 y la asociación con alteraciones psicológicas, ya que dependerá en gran medida el curso de la enfermedad, la existencia de redes de apoyo para que se desencadene en resultados positivos o negativos, que se manifiestan en el autocuidado. Se ha demostrado con anterioridad que fortalecer el apoyo social en estos pacientes, puede mejorar los factores psicológicos, y que por consiguiente vamos a tener resultados globales positivos, como la adherencia adecuada al tratamiento farmacológico y una correcta alimentación, entre otros (Aflakseir \& Malekpour, 2014; Beléndez, 2015; Harandi, Taghinasab, \& Nayeri, 2017; Williams et al., 2016). El apoyo social es importante para ayudar al paciente con diabetes a sobrellevar la enfermedad y para mejorar la adherencia al tratamiento. Los proveedores de atención médica deben tener en cuenta los factores psicosociales en el régimen de tratamiento del paciente, así como incluir a los miembros de la familia, para que sean educados sobre la diabetes, la importancia en el apoyo para la adherencia y las complicaciones a largo plazo de la enfermedad (Ramkisson, 2017).

El apoyo social juega un papel fundamental en los pacientes con diabetes y es importante para que puedan hacer frente de manera efectiva a la enfermedad. Estos hallazgos nos proporcionan elementos clave en los que debemos centrarnos para la atención de adultos mayores con DM2, especialmente en el área de apoyo social y salud mental, e indican la necesidad de continuar explorando el fenómeno de la relación entre DM2 y Estado emocional.

\section{OBJETIVO DE LA INVESTIGACIÓN}

El objetivo de este trabajo fue explorar la influencia del riesgo social en la adherencia terapéutica al tratamiento farmacológico en pacientes con DM2 mayores de 65 años.

\section{MUESTRA Y/O PARTICIPANTES}

La población del estudio abarcó a todas las personas mayores de 65 años, asignadas a los centros de salud de la Dirección Asistencial Norte de Madrid, diagnosticadas, mediante la Clasificación Internacional de Atención Primaria (CIAP-2) con diabetes Mellitus tipo 2 (código T90) (N=26703)(WONCA, 1999). La ventana temporal en la que extrajeron los datos fue en el año 2015.

La Dirección Asistencial Norte presenta un nivel socioeconómico heterogéneo. Se caracteriza por ser una de las zonas de la comunidad de Madrid que abarca distritos con una concentración importante de población envejecida con una proporción del 15,6\% (hombres: 11,6\%, mujeres: 18,5\%) y un índice de envejecimiento de 80,5 para hombres y 115,6 para mujeres respectivamente, siendo de 97,5 para toda la Dirección (2016)(Primaria, 2016).

\section{METODOLOGÍA Y/O INSTRUMENTOS UTILIZADOS}

DISEÑO

Estudio descriptivo transversal.

VARIABLES E INSTRUMENTOS PARA LA RECOGIDA DE LOS DATOS

Todas las variables fueron extraídas de la Historia Clínica Electrónica de Atención Primaria de la Comunidad de Madrid, Sistema de Información AP-Madrid.

Variables dependientes:

-Adherencia al tratamiento farmacológico: se categorizó la variable en Sí/No, atendiendo la clasificación al Test de Morisky Green(Val Jiménez A, Amorós Ballestero G, Martínez Visa P, Fernández Ferré ML, 1992), que valora si el paciente adopta actitudes correctas en relación al tratamiento. Esta variable se consideró también como variable independiente para el control de la DM2. 
Variables independientes

* Clínicas: Diagnóstico médico para la depresión o depresión maniaca (CIAP-P73;P76)(Wonca, 1999), Diagnóstico médico para la ansiedad, crisis de ansiedad, (CIAP-P01;P74)(Wonca, 1999); Clínica (síntomas de depresión y ansiedad) (Sí/No; se pregunta al paciente si ha tenido alguna alteración del estado emocional); riesgo social (Cimbage et al., 2014), que incluyen preguntas como redes directas e indirectas de apoyo, como familiares, amigos, compañeros, etc., problemas de adaptación personal, los cuales incluyen preguntas como cambios importantes, estrés, probable adaptación, participación en la toma de decisiones, etc., (Si/No) en base a las respuestas afirmativas se deduce la presencia o no de problemas de alteración mental; Tiempo desde el diagnóstico de diabetes, ansiedad y depresión (en años).

*Sociodemográficas: Zona de origen (categorizada en regiones según el país de procedencia en: América centro y sur, África, Asia, Europa del este, países de la OCDE, España y otros); Edad (en años); Sexo; Zona de residencia (según la zona básica de salud de la Dirección Asistencial Norte a la que estuviese asignado el paciente); Situación socioeconómica de la zona de residencia, medida a través del índice de privación (IP) (DominguezBerjón MF, Borrel C, Cano-Serral G, Esnaola S, Nolasco A, 2008) de la zona básica de salud. Para este trabajo se utilizó el IP con datos del 2011 (proporcionado por los creadores del índice) y se operacionalizó en cuartiles, siendo el cuartil 1 el grupo con mejor situación y el cuartil 4 el grupo con peor situación socioeconómica (Débora Álvarez-delArco, MartaVicente Sánchez, BelénAlejos, 2013).

Variables potencialmente confusoras/modificadoras de efecto:

Sobrepeso/Obesidad (IMC) (categorizada según la clasificación de la OMS, normopeso, sobrepeso, obesidad)(OMS, 2018); Actividad física (Si actividad física/No actividad física); Adherencia al régimen dietético (Si cumple con el régimen dietético/No cumple con el Régimen Dietético).

Tratamiento y análisis de datos:

Se calcularon los índices de estadística descriptiva en función de la naturaleza de las variables (media y desviación estándar -DE- para las cuantitativas, y frecuencias absolutas y porcentajes para las cualitativas). Se realizaron análisis bivariantes para cada una de las variables independientes con las dependientes. Para el contraste de hipótesis con variables cualitativas se utilizó la prueba de Ji Cuadrado y para variables cuantitativas la t de Student y ANOVA. Se calcularon prevalencias de mal control de la DM y de No adherencia al tratamiento farmacológico. Se efectuaron análisis multivariante de regresión logística para las variables dependientes (Control de DM2 y Adherencia Terapéutica al Tratamiento

Farmacológico); con el fin de disminuir la probabilidad de no incluir en el modelo final algún factor relevante, se incluyeron en el análisis aquellas variables asociadas con el control de DM y adherencia al tratamiento a un nivel bivariante con una $p \leq 0,20$ (Ayçaguer, 1997). Para el análisis estadístico, se utilizó el programa Stata 12.0.

Consideraciones éticas:

Se solicitó autorización para la realización del estudio al Comité de Ética de la Universidad Autónoma de Madrid, así como de la Comisión Central de Investigación de la Gerencia Asistencial de Atención Primaria de Madrid. El acceso de las historias clínicas contenidas en dicha base de datos está regulado por las consideraciones éticas referentes al artículo 8 sobre datos relativos a la salud de la Ley Orgánica 15/1999 de protección de datos de carácter personal, y en la ley 14/1986, General de Sanidad.

\section{RESULTADOS}

Se obtuvo información de 26703 sujetos de 65 años y más que cumplían con el criterio de diagnóstico de DM2. El porcentaje de mujeres fue de $51,1 \%$ ( $n=13639)$. La edad media fue de 77,4 años ( $D E=7,59)$. Un $94,90 \%$ $(n=12944)$ de las mujeres y un $95,2 \%$ ( $n=12443)$ de los hombres eran autóctonos/as. En lo referente al IMC, se encontró que un 46,7\% ( $n=3428$ ) tenía algún grado de obesidad en mujeres, y un 40,4\% (n=2770) en los hombres. Existía un mayor porcentaje de diagnóstico en depresión en mujeres (23,8\%, n=3248) frente a los hombres (9,3\% n=1214); así como de diagnóstico de ansiedad (mujeres 22,2\%, $n=3029$; hombres 10\% $n=1307)$. En cuanto a la situación socioeconómica (índice de privación) la mayoría de la población de la dirección asistencial 
norte corresponde al cuartil 3 (mujeres 30,7\%, $n=4188$; hombres 28,2\%, $n=3688$ ). El tiempo del diagnóstico medio de la DM2 fue de 10,2 años (DE=6,75) (tabla 1). 
Tabla 1: Descripción de la población de estudio según sexo

\begin{tabular}{|c|c|c|c|c|}
\hline Variable & Categorías & $\begin{array}{l}\text { Mujeres } \\
(n=13639) \\
\%(\#)\end{array}$ & $\begin{array}{l}\text { Hombres } \\
(n=13064) \\
\%(\#)\end{array}$ & $\begin{array}{l}\text { Total } \\
n=26703 \\
\%(\#)\end{array}$ \\
\hline \multirow[t]{2}{*}{ Sexo } & Hombre & & & 48,9 (13064) \\
\hline & Mujer & & & $51,1 \quad(13639)$ \\
\hline \multirow[t]{9}{*}{ Región de Procedencia } & América centro y & $1,5(206)$ & $1,5(197)$ & $1,5(403)$ \\
\hline & sur & $0,8(112)$ & $1,9(121)$ & $0,9(233)$ \\
\hline & África & $0,8(109)$ & $0,7(93)$ & $0,8(202)$ \\
\hline & Asia & $0,8(104)$ & $0,9 \quad 113)$ & $0,8(217)$ \\
\hline & Países de la & $0,3(41)$ & 0,1 & $0,2(58)$ \\
\hline & OCDE & 94,9 & 95,3 & $95,1(25387)$ \\
\hline & Europa del Este & (12944) & (12443) & $0,8 \quad(203)$ \\
\hline & España & $0,9 \quad(123)$ & $0,6 \quad(80)$ & \\
\hline & Otros & & & \\
\hline \multirow[t]{8}{*}{ Índice de Privación } & Cuartil 1 & 21,5 & $25,3(3311)$ & $23,4(6238)$ \\
\hline & Cuartil 2 & (2927) & $24,8(3240)$ & $24,7(6587)$ \\
\hline & Cuartil 3 & 24,5 & $28,2(3688)$ & $29,5(7876)$ \\
\hline & Cuartil 4 & $(3347)$ & $21,6(2825)$ & $22,5(6002)$ \\
\hline & & 30,7 & & \\
\hline & & (4188) & & \\
\hline & & 23,3 & & \\
\hline & & (3177) & & \\
\hline \multirow[t]{4}{*}{ Índice de Masa corporal } & Normopeso & 16,1 & $13,6(931)$ & $14,9(2114)$ \\
\hline & Sobrepeso & (1183) & $46,1(3162)$ & $41,5(5898)$ \\
\hline & Obesidad & $\begin{array}{l}37,2 \\
(2736)\end{array}$ & $40,4(2770)$ & $43,6(6198)$ \\
\hline & & $\begin{array}{l}46,7 \\
(3428)\end{array}$ & & \\
\hline \multirow[t]{3}{*}{ Ejercicio Físico } & Sí & 69,2 & $84,5(4180)$ & $76,5(7941)$ \\
\hline & No & (3761) & $15,5(766)$ & $23,5(2437)$ \\
\hline & & $\begin{array}{l}30,8 \\
(1671)\end{array}$ & & \\
\hline \multirow[t]{3}{*}{ Adherencia al régimen dietético } & Sí & 18,0 & $19,0(2477)$ & $18,5(4927)$ \\
\hline & No & $(2450)$ & 81,0 & $81,5(21776)$ \\
\hline & & $\begin{array}{l}82,0 \\
(11189)\end{array}$ & $(10587)$ & \\
\hline \multirow[t]{5}{*}{ Tratamiento farmacológico } & $\mathrm{ADO}$ & 13,4 & 14,7 (1917) & $14,0(3739)$ \\
\hline & Insulina & $(1822)$ & $1,2(161)$ & $1,3(355)$ \\
\hline & ADO+insulina & $1,4(194)$ & $3,5(451)$ & $3,5(932)$ \\
\hline & Ninguno & $3,5(481)$ & 80,6 & $81,2(21677)$ \\
\hline & & $\begin{array}{l}81,7 \\
(11142)\end{array}$ & $(10535)$ & \\
\hline \multirow[t]{2}{*}{ Riesgo Social } & Sí & 31,5 & $17,1(564)$ & $25,6(2080)$ \\
\hline & No & $\begin{array}{l}(1516) \\
68,5 \\
(3293)\end{array}$ & $82,9(2735)$ & $74,3(6028)$ \\
\hline
\end{tabular}




\begin{tabular}{|c|c|c|c|c|}
\hline adaptación & Sí & $14,8(282)$ & $8,6(154)$ & $77,8(436)$ \\
\hline personal & No & $\begin{array}{l}85,3 \\
(1630)\end{array}$ & $91,9(1640)$ & $88,2(3270)$ \\
\hline $\begin{array}{l}\text { Control de la diabetes Mellitus } \\
\text { tipo } 2\end{array}$ & $\begin{array}{l}\text { Buen control } \\
\text { Mal control } \\
\text { No registrado }\end{array}$ & $\begin{array}{l}36,5 \\
(4980) \\
18,9 \\
(2571) \\
44,6 \\
(6088)\end{array}$ & $\begin{array}{l}34,3(4476) \\
17,2(2246) \\
48,6(6342)\end{array}$ & $\begin{array}{l}35,4(9456) \\
18,0(4817) \\
46,6(12430)\end{array}$ \\
\hline $\begin{array}{l}\text { Adherencia al tratamiento } \\
\text { farmacológico }\end{array}$ & $\begin{array}{l}\text { Sí } \\
\text { No } \\
\text { No registrado }\end{array}$ & $\begin{array}{l}31,8 \\
(4333) \\
1,0(135) \\
67,2 \\
(9171)\end{array}$ & $\begin{array}{l}22,6(2950) \\
0,7(95) \\
76,7 \\
(10019)\end{array}$ & $\begin{array}{l}27,3(7283) \\
0,9(230) \\
71,9(19190)\end{array}$ \\
\hline Diagnóstico de Depresión & $\begin{array}{l}\text { Sí } \\
\text { No }\end{array}$ & $\begin{array}{l}23,8 \\
(3248) \\
76,2 \\
(10391)\end{array}$ & $\begin{array}{l}9,3(1214) \\
90,7 \\
(11850)\end{array}$ & $\begin{array}{l}16,7(4462) \\
83,3(22241)\end{array}$ \\
\hline Diagnóstico de Ansiedad & $\begin{array}{l}\text { Sí } \\
\text { No }\end{array}$ & $\begin{array}{l}22,2 \\
(3029) \\
77,8 \\
(10610)\end{array}$ & $\begin{array}{l}10,0(1307) \\
90,0 \\
(11757)\end{array}$ & $\begin{array}{l}16,2(4336) \\
83,8(22367)\end{array}$ \\
\hline Clínica de Depresión & $\begin{array}{l}\text { Sí síntomas } \\
\text { No síntomas }\end{array}$ & $\begin{array}{l}38,2(120) \\
61,8(194)\end{array}$ & $\begin{array}{l}34,3(36) \\
65,7(69)\end{array}$ & $\begin{array}{l}37,2(159) \\
62,8(263)\end{array}$ \\
\hline Clínica de Ansiedad & $\begin{array}{l}\text { Sí síntomas } \\
\text { No Síntomas }\end{array}$ & $\begin{array}{l}27,1(211) \\
72,9(569)\end{array}$ & $\begin{array}{l}18,1(69) \\
81,9(312)\end{array}$ & $\begin{array}{l}24,1(280) \\
75,9(881)\end{array}$ \\
\hline & & $\begin{array}{l}\text { Media } \\
\text { (DE) }\end{array}$ & $\begin{array}{l}\text { Media } \\
\text { (DE) }\end{array}$ & Media (DE) \\
\hline \multicolumn{2}{|c|}{ Edad (años) } & $78,80(7,8)$ & $75,94(7,1)$ & $77,40(7,6)$ \\
\hline \multicolumn{2}{|c|}{ Tiempo de diagnóstico de diabetes (años) } & $10,40(6,9)$ & $10,06(6,6)$ & $10,24(6,8)$ \\
\hline \multirow{2}{*}{\multicolumn{2}{|c|}{$\begin{array}{l}\text { Tiempo de diagnóstico de ansiedad (años) } \\
\text { Tiempo de diagnóstico de depresión (años ) }\end{array}$}} & $7,91(4,4)$ & $7,38(4,4)$ & $7,75(4,4)$ \\
\hline & & $9,25(5,8)$ & $7,7(5,3)$ & $8,8(5,7)$ \\
\hline
\end{tabular}

OCDE: Organización para la Cooperación y Desarrollo Económico; ADO: Antidiabéticos Orales DE: Desviación Estándar.

El 97,0\% ( $n=4333$ ) de las mujeres y el 96,9\% ( $n=2950)$ de los hombres presentaron adherencia al tratamiento farmacológico (tabla 2).

Tabla 2: Prevalencia de No Adherencia al tratamiento farmacológico

\begin{tabular}{|ccccccc|}
\hline $\begin{array}{l}\text { Global } \\
\%(\#)\end{array}$ & IC95\% & $\begin{array}{l}\text { Mujeres } \\
\%(\#)\end{array}$ & IC95\% & $\begin{array}{l}\text { Hombres } \\
\%(\#)\end{array}$ & IC95\% \\
No adherencia & $3,06(230)$ & $2,67-3,45$ & $3,02(135)$ & $2,52-3,52$ & $3,12(95)$ & $2,5-3,74$ \\
\hline
\end{tabular}

IC95\%: Intervalo de confianza al $95 \%$ de seguridad

\section{No adherencia al tratamiento farmacológico}

Las mujeres de origen extranjero tenían mayores prevalencias de no adherencia al tratamiento farmacológico (No AdhT) que las de origen español/OCDE (9,8\% vs 2,9\%; $p=0,002)$. En lo que respecta al índice de privación, se observó que a medida que baja el nivel socioeconómico del barrio aumentaba la prevalencia de No AdhT, especialmente en los hombres, aunque no se había significación estadística (tabla 3). 


\section{RIESGO SOCIAL COMO FACTOR DE RIESGO PARA LA NO ADHERENCIA FARMACOLÓGICA EN}

PACIENTES CON DM2 MAYORES DE 65 AÑOS

La prevalencia de No AdhT fue mayor en quienes no hacían ejercicio, tanto en mujeres (5,5\% vs 1,9\%; $p=0,000)$ como en hombres (5,8\% vs 2,4\%; $P=0,001)$. Tener riesgo social también se asoció a mayores prevalencias de NO AdhT (Mujeres: 5,7\% vs 1,9\%; $p=0,000$. Hombres: 7,5\% vs 2,3\%; $p=0,000$ ). Respecto al tratamiento farmacológico, en el caso de las mujeres, se daban prevalencias de № AdhT más altas cuando había tratamiento con insulina $(\mathrm{p}=0,044)$. También las mujeres que no tenían adherencia al régimen dietético, tenían mayores prevalencias de № AdhT (3,4\% vs $1,7 \%$; $p=0,006)$ (tabla 3 ).

Se asoció a mayor prevalencia de No AdhT tener diagnóstico de depresión en las mujeres (3,8\% vs 2,7\%; $\mathrm{p}=0,055)$; y en los hombres tener diagnóstico de ansiedad ( $6,8 \%$ vs $3 \% ; \mathrm{p}=0,043)$, así como tener clínica de depresión (15\% vs 1,9\%; p=0,028) (tabla 3). 
Tabla 3: Prevalencia de no adherencia al tratamiento según variables sociodemográficas y clínicas

\begin{tabular}{|c|c|c|c|c|c|c|c|}
\hline Variables & Categorías & $\begin{array}{l}\text { Mujeres } \\
\% \text { (\#) }\end{array}$ & $\begin{array}{l}\text { Valor } \\
\text { p }\end{array}$ & $\begin{array}{l}\text { Hombres } \\
\% \text { (\#) }\end{array}$ & $\begin{array}{l}\text { Valor } \\
\text { p }\end{array}$ & $\begin{array}{l}\text { Total } \\
\%(\#)\end{array}$ & $\begin{array}{l}\text { Valor } \\
\text { p* }^{*}\end{array}$ \\
\hline $\begin{array}{ll}\text { Región } & \text { de } \\
\text { procedencia }\end{array}$ & $\begin{array}{l}\text { América centro } \\
\text { y sur } \\
\text { África } \\
\text { Asia } \\
\text { Países(OCDE) } \\
\text { Europa del este } \\
\text { España } \\
\text { Otros }\end{array}$ & $\begin{array}{l}- \\
9,1 \quad(2) \\
25,0(2) \\
- \\
- \\
2,9 \\
(129) \\
15,4(2)\end{array}$ & 0,000 & $\begin{array}{l}- \\
- \\
10,0(1) \\
3,6(1) \\
- \\
3,14(93) \\
-\end{array}$ & 0,674 & $\begin{array}{l}- \\
5,4 \\
(2) \\
16,7 \\
(3) \\
1,9 \\
(1) \\
- \\
3,0 \\
(222) \\
8,7 \\
(2)\end{array}$ & 0,014 \\
\hline $\begin{array}{ll}\text { Región } & \text { de } \\
\text { procedencia } & \end{array}$ & $\begin{array}{l}\text { Origen } \\
\text { extranjero } \\
\text { España+OCDE }\end{array}$ & $\begin{array}{l}9,8 \quad(6) \\
2,9 \\
(129)\end{array}$ & 0,002 & $\begin{array}{ll}1,7 & (1) \\
3,1 & (94)\end{array}$ & 0,525 & $\begin{array}{l}5,8 \\
(7) \\
3,0 \\
(223)\end{array}$ & 0,076 \\
\hline $\begin{array}{l}\text { Índice } \\
\text { Privación }\end{array}$ & $\begin{array}{l}\text { Cuartil } 1 \\
\text { Cuartil } 2 \\
\text { Cuartil } 3 \\
\text { Cuartil } 4\end{array}$ & $\begin{array}{ll}2,3 & (24) \\
3,7 & (36) \\
3,1 & (45) \\
2,9 & (30)\end{array}$ & 0,359 & $\begin{array}{ll}4,0 & (33) \\
3,0 & (20) \\
3,4 & (31) \\
1,7 & (11)\end{array}$ & 0,073 & $\begin{array}{l}3,1 \\
(57) \\
3,4 \\
(56) \\
3,3 \\
(76) \\
2,5 \\
(41)\end{array}$ & 0,392 \\
\hline IMC & $\begin{array}{l}\text { Normopeso } \\
\text { Sobrepeso } \\
\text { Obesidad }\end{array}$ & $\begin{array}{ll}2,9 & (15) \\
2,1 & (24) \\
3,6 & (48)\end{array}$ & 0,091 & $\begin{array}{ll}3,4 & (11) \\
2,7 & (26) \\
3,7 & (29)\end{array}$ & 0,481 & $\begin{array}{l}3,1 \\
(26) \\
2,4 \\
(50) \\
3,6 \\
(76)\end{array}$ & 0,058 \\
\hline Ejercicio físico & $\begin{array}{l}\text { Sí } \\
\text { No }\end{array}$ & $\begin{array}{l}1,9(30) \\
4,5(41)\end{array}$ & 0,000 & $\begin{array}{l}2,4(31) \\
5,8(19)\end{array}$ & 0,001 & $\begin{array}{l}2,1 \\
(61) \\
4,8 \\
(60)\end{array}$ & 0,000 \\
\hline Riesgo social & $\begin{array}{l}\text { Sí } \\
\text { No }\end{array}$ & $\begin{array}{l}5,7(68) \\
1,9(53)\end{array}$ & 0,000 & $\begin{array}{ll}7,5 & (33) \\
2,3 & (54)\end{array}$ & 0,000 & $\begin{array}{l}6,1 \\
(101) \\
2,1 \\
(107)\end{array}$ & 0,000 \\
\hline
\end{tabular}




\begin{tabular}{|c|c|c|c|c|c|c|c|}
\hline $\begin{array}{l}\text { Problemas de } \\
\text { adaptación } \\
\text { personal }\end{array}$ & $\begin{array}{l}\text { Sí } \\
\text { No }\end{array}$ & $\begin{array}{l}4,2(5) \\
2,6(19)\end{array}$ & 0,342 & $\begin{array}{l}4,4(2) \\
1,5(8)\end{array}$ & 0,136 & $\begin{array}{l}4,3(7) \\
2,1 \\
(27)\end{array}$ & 0,091 \\
\hline $\begin{array}{l}\text { Tratamiento } \\
\text { farmacológico }\end{array}$ & $\begin{array}{l}\text { ADO } \\
\text { Insulina } \\
\text { ADO + Insulina } \\
\text { Ninguno }\end{array}$ & $\begin{array}{l}2,0(13) \\
4,6(4) \\
0,8(2) \\
3,3 \\
(116)\end{array}$ & 0,044 & $\begin{array}{l}2,6(13) \\
2,0(1) \\
4,1(7) \\
3,2(74)\end{array}$ & 0,739 & $\begin{array}{l}2,2 \\
(26) \\
3,7(5) \\
2,2(9) \\
3,3 \\
(190)\end{array}$ & 0,194 \\
\hline $\begin{array}{l}\text { Adherencia al } \\
\text { régimen dietético }\end{array}$ & $\begin{array}{l}\text { Sí } \\
\text { No }\end{array}$ & $\begin{array}{l}1,7 \quad(16) \\
3,4 \\
(119)\end{array}$ & 0,006 & $\begin{array}{l}3,0(21) \\
3,2(74)\end{array}$ & 0,818 & $\begin{array}{l}2,2 \\
(37) \\
3,3 \\
(193)\end{array}$ & 0,025 \\
\hline $\begin{array}{l}\text { Diagnóstico de } \\
\text { depresión }\end{array}$ & $\begin{array}{l}\text { Sí } \\
\text { No }\end{array}$ & $\begin{array}{ll}3,8 & (47) \\
2,7 & (88)\end{array}$ & 0,055 & $\begin{array}{ll}3,7 & (13) \\
3,1 & (82)\end{array}$ & 0,532 & $\begin{array}{l}3,8 \\
(60) \\
2,9 \\
(170)\end{array}$ & 0,060 \\
\hline $\begin{array}{l}\text { Diagnóstico de } \\
\text { ansiedad }\end{array}$ & $\begin{array}{l}\text { Sí } \\
\text { No }\end{array}$ & $\begin{array}{l}1,7 \quad(4) \\
3,1 \\
(131)\end{array}$ & 0,240 & $\begin{array}{l}6,8(6) \\
3,0 \quad(89)\end{array}$ & 0,043 & $\begin{array}{l}3,1 \\
(10) \\
3,1 \\
(220)\end{array}$ & 0,938 \\
\hline $\begin{array}{l}\text { Clínica de } \\
\text { depresión }\end{array}$ & $\begin{array}{l}\text { Sí } \\
\text { No }\end{array}$ & $\begin{array}{l}4,8(4) \\
3,7(5)\end{array}$ & 0,693 & $\begin{array}{l}15,0(3) \\
1,9 \quad(1)\end{array}$ & 0,028 & $\begin{array}{l}6,7(7) \\
3,2(6)\end{array}$ & 0,157 \\
\hline $\begin{array}{ll}\text { Clínica } & \text { de } \\
\text { ansiedad } & \end{array}$ & $\begin{array}{l}\text { Sí } \\
\text { No }\end{array}$ & $\begin{array}{l}1,6(1) \\
1,8(3)\end{array}$ & 0,933 & $\begin{array}{l}12,5(2) \\
5,6 \quad(4)\end{array}$ & 0,319 & $\begin{array}{ll}3,9 & (3) \\
2,9 & (7)\end{array}$ & 0,678 \\
\hline $\begin{array}{l}\text { Edad del } \\
\text { paciente (años) }\end{array}$ & $\begin{array}{l}\text { Sí AdhT } \\
\text { No AdhT }\end{array}$ & $\begin{array}{l}\text { Media } \\
\text { (DE) } \\
83,5 \\
(5,41) \\
83,3 \\
(5,29)\end{array}$ & 0,707 & $\begin{array}{l}\text { Media } \\
\text { (DE) } \\
83,1(5,0) \\
80,9 \\
(5,12)\end{array}$ & 0,029 & $\begin{array}{l}\text { Media } \\
\text { (DE) } \\
82,9 \\
(5,31) \\
82,3 \\
(5,34)\end{array}$ & 0,098 \\
\hline $\begin{array}{l}\text { Tiempo de } \\
\text { diagnóstico de } \\
\text { diabetes (años) }\end{array}$ & $\begin{array}{l}\text { Sí AdhT } \\
\text { No AdhT }\end{array}$ & $\begin{array}{l}11,8 \\
(7,23) \\
12,4 \\
(9,11)\end{array}$ & 0,335 & $\begin{array}{l}11,5 \\
(7,14) \\
13,0 \\
(11,15) \\
\end{array}$ & 0,053 & $\begin{array}{l}11,7 \\
(7,19) \\
12,7 \\
(9,98) \\
\end{array}$ & 0,048 \\
\hline
\end{tabular}

*entre mujeres y hombres; DE: Desviación Estándar; AdhT: adherencia al tratamiento farmacológico

Atendiendo a los modelos de regresión logística múltiple, tener riesgo social (Modelo global: OR: 4,97, IC95\%: 2,01-12,3; Modelo en mujeres: OR: 2,92, IC95\%: 1,61-5,3; Modelo en hombres: OR: 3,52 IC95\%: 1,96,52), tener depresión (Modelo global: OR: 3,01, IC95\%: 1,23-7,36), síntomas de ansiedad (Modelo en hombres: 
OR: 2,57 IC95\%: 1,21-5,45) y vivir en una zona de menor nivel socioeconómico ( Modelo en mujeres: OR: 3,15, IC95\%: 1,18-8,39) aumenta el riesgo de no cumplir con el tratamiento farmacológico (tabla 4).

Tabla 4: Modelos multivariante para la no adherencia al tratamiento farmacológico

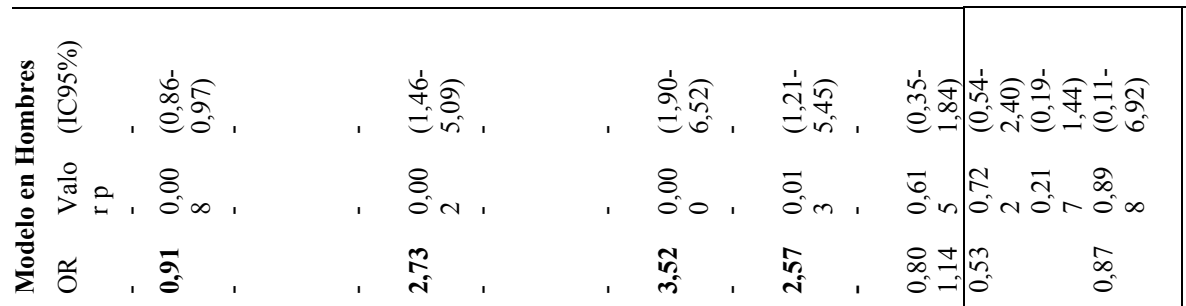

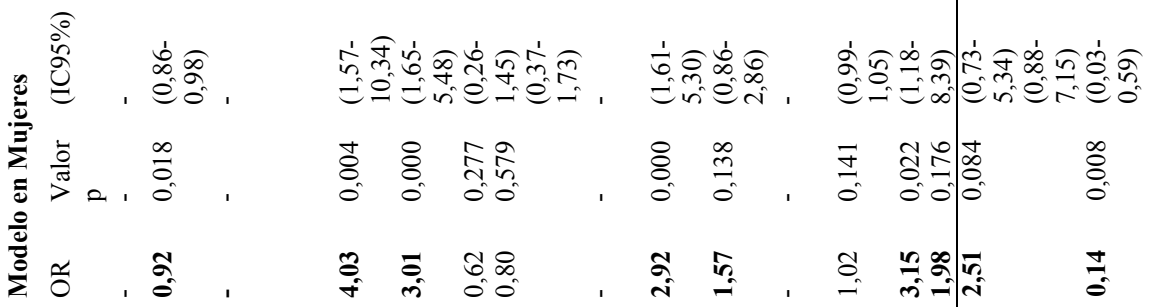

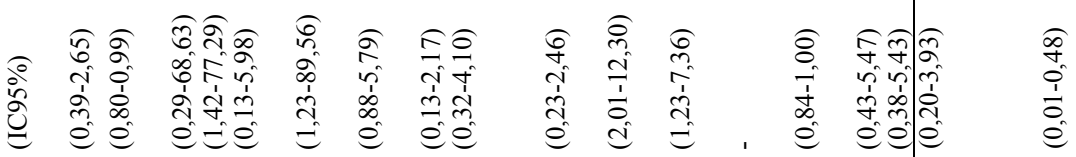

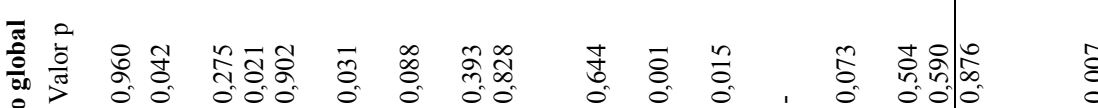

这

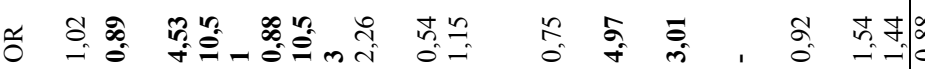

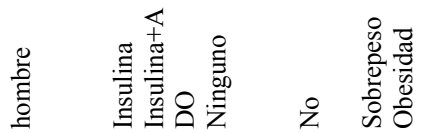

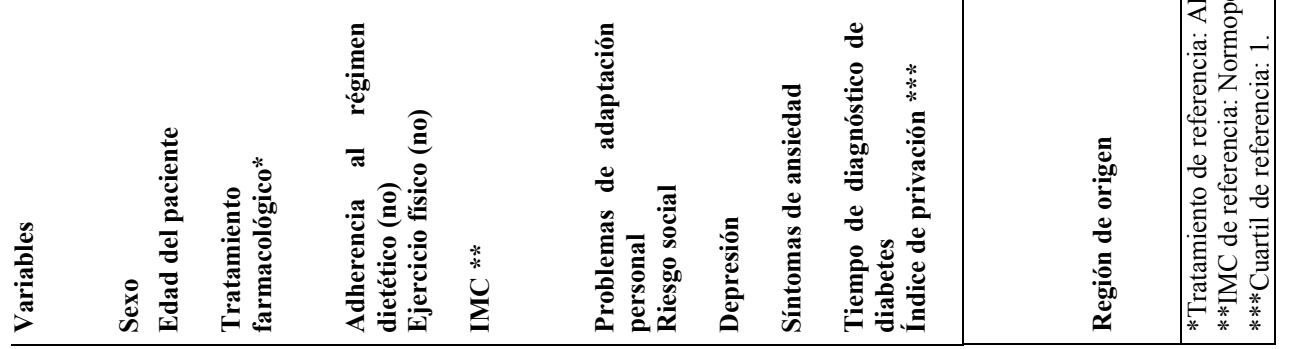

OCDE: Organización para la Cooperación y Desarrollo Económico; ADO: Antidiabéticos Orales; IC95\%: Intervalo de Confianza al 95\% de Seguridad 


\section{RIESGO SOCIAL COMO FACTOR DE RIESGO PARA LA NO ADHERENCIA FARMACOLÓGICA EN PACIENTES CON DM2 MAYORES DE 65 AÑOS}

\section{DISCUSIÓN Y CONCLUSIONES}

En el presente estudio las prevalencias de mal control de DM2 en las personas mayores de 65 años se elevan a medida que disminuye el nivel socioeconómico de la zona de residencia, y también en las personas de origen extranjero, lo que pone de manifiesto la existencia de desigualdades sociales en salud entorno a la aparición, progreso y manejo de la DM, que ya han sido descritas en la literatura y advierten que quienes tienen peor situación socioeconómica, tienen cifras de control más desfavorables(Espelt et al., 2013)(Soler-González et al., 2013)(Parro Moreno A, Santiago Pérez IM, Abraira V, Aréjula Torres JL, Gandarillas-Grande A, Díaz Holgado A, Morales Asencio JM, 2016).

Por otra parte, también se encontró una mayor prevalencia de no AdhT en personas de origen extranjero, 10 que también puede revelar la existencia de estas desigualdades en salud; Soler-González et al (Soler-González et al., 2013) encontraron un peor registro de los indicadores de calidad en la atención de la diabetes mellitus en algunos grupos de población inmigrante, lo que podría estar evidenciando desigualdades en la atención sanitaria, si bien hay factores como las características físicas, el tipo de dieta y actividad física realizada que también pueden influir de manera diferente en esta población (DL, C, 2010).

Nuestros resultados reflejan que tener riesgo social y problemas de adaptación personal influyen negativamente en la adherencia al tratamiento lo que está en consonancia con lo que muestran otros trabajos que indican que el aislamiento social, es decir, la falta de redes de apoyo como la familia y amigos son factores de riesgo que se van acumulando a lo largo de toda la vida, y se manifiestan de forma negativa especialmente en pacientes crónicos adultos mayores, aumentando la posibilidad de déficit del autocuidado particularmente en lo que tiene que ver con el tratamiento farmacológico (Smalls et al., 2012)(Aflakseir \& Malekpour, 2014)(Harandi et al., 2017)(Maheri et al., 2018).

Sumado a lo anteriormente mencionado, entre las principales limitaciones que presenta este estudio, se encuentran las derivadas del uso de fuentes documentales, que especialmente en el caso de la medición de la adherencia terapéutica, así como en los problemas de salud mental, pusieron de manifiesto un gran infraregistro.

Una de las fortalezas de la presente investigación es que se ha abordado toda la población de un área asistencial, lo que garantiza la elevada representatividad de la población objeto de estudio, además de una mayor precisión de las estimaciones, y finalmente contribuye a una mayor validez externa.

En conclusión, los hallazgos obtenidos permiten demostrar una asociación de los problemas relacionados al aislamiento social con la no adherencia al tratamiento farmacológico en pacientes DM2 mayores de 65 años, además se pone de manifiesto que el riesgo social se observó como un factor de riesgo para la no AdhT, así como los síntomas de ansiedad y depresión en los hombres.

Los resultados obtenidos indican la necesidad de seguir profundizando en este tema, y abordarlo desde diferentes enfoques como sería el que ofrece el paradigma cualitativo.

\section{REFERENCIAS BIBLIOGRÁFICAS}

Aflakseir, A., \& Malekpour, F. (2014). The Role of Self-Efficacy and Social Support in Predicting Depression Symptoms in Diabetic Patients. Iranian Journal of Diabetes and Obesity, 6(3).

Axon, R. N. (2016). Comorbid depression is differentially depression is differentially associated with longitudinal medication nonadherence by race/ethnicity in patients with type 2 diabetes. Medicine, 30(95), 1-7.

Ayçaguer, S. (1997). Cultura estadística e investigación científica en el campo de la salud: una mirada crítica. Madrid: Diaz Santos.

Basterra-Gortari FJ, B.-R. M. et al. (2017). Prevalencia de obesidad y diabetes en adultos españoles, 1987-2012. Medicina Clínica, 148(6), 250-256. https://doi.org/10.1016/j.medcli.2016.11.022

Beléndez, M. (2015). Estrés emocional y calidad de vida en personas con diabetes y sus familiares. Gaceta Sanitaria, 29(4), 300-303. 
Cho, N. H., Shaw, J. E., Karuranga, S., Huang, Y., Rocha, J. D., Ohlrogge, A. W., \& Malanda, B. (2018). IDF Diabetes Atlas: Global estimates of diabetes prevalence for 2017 and projections for 2045. Diabetes Research and Clinical Practice, 138, 271-281. https://doi.org/10.1016/j.diabres.2018.02.023

Cimbage, C., Thomasz, 0., Caruana, C., Eugenia, M., Miguel, J., Thomasz, E. 0., ... Miguel, J. (2014). Riesgo social: medición de la vulnerabilidad en grupos focalizados. Cuadernos del CIMBAGE, 16, $27-51$.

Débora Álvarez-delArco, MartaVicente Sánchez, BelénAlejos, C. P. y E. R. (2013). Construcción de un índice de privación para los barrios de Madrid y Barcelona. Revista Española de Salud Pública, 87, 317-329.

DL, C. (2010). Moving to an A1C-Based Diagnosis of Diabetes Has a Different Impact on Prevalence in Different Ethnic Groups. Diabetes Care, 33(3), 580-582.

Dominguez-Berjón MF, Borrel C, Cano-Serral G, Esnaola S, Nolasco A, P. M. (2008). Construcción de un índice de privación a partir de datos censales en grandes ciudades españolas (Proyecto MEDEA). Gaceta Sanitaria, 22(3), 179-187.

Espelt, A., Borrell, C., Palència, L., Goday, A., Spadea, T., Gnavi, R., ... Kunst, A. E. (2013). Socioeconomic inequalities in the incidence and prevalence of type 2 diabetes mellitus in Europe. Gaceta Sanitaria, 27(6), 494-501. https://doi.org/10.1016/j.gaceta.2013.03.002

Funnell, M. (2006). The Diabetes Attitudes, Wishes and Needs (DAWN) study. Clinical Diabetes, 4(24), 154-155.

Gandarillas Ana María, Valero Virginia, G. M. (2018). Estudio PREDIMERC 2015 PREvalencia de Dlaberes MEllitus y Riesgo Cardiovascular en población adulta de la Comunidad de Madris. Madrid.

Garcia, M. L., Castañeda, S. F., Allison, M. A., Elder, J. P., \& Talavera, G. A. (2019). Correlates of Low-adherence to Oral Hypoglycemic Medications among Hispanic/Latinos of Mexican Heritage with Type 2 Diabetes in the United States. Diabetes Research and Clinical Practice, 18(31), 644-649. https://doi.org/10.1016/j.diabres.2019.04.007

Harandi, T. F., Taghinasab, M. M., \& Nayeri, T. D. (2017). The correlation of social support with mental health: A meta-analysis. Electronic Physician, (September), 5212-5222.

Joseph, J. J., \& Golden, S. H. (2018). Cortisol dysregulation: the bidirectional link between stress, depression, and type 2 diabetes mellitus. Annals of the New York Academy of Sciences, 1391(1), 20-34. https://doi.org/10.1111/nyas.13217.Cortisol

Joshep JJ, G. S. (2017). Cortisol dysregulation: the bidirectional link between stress, depression, and type 2 diabetes mellitus. Annals of the New York Academy of Sciences, 1(1391), 20-34.

Koetsenruijter, J., Eikelenboom, N. Van, Lieshout, J. Van, Vassilev, I., Lionis, C., Todorova, E., ... Wensing, M. (2016). Social support and self-management capabilities in diabetes patients : An international observational study. Patient Education and Counseling, 99(4), 638-643. https://doi.org/10.1016/j.pec.2015.10.029

Maheri, A., Sadeghi, R., Shojaeizadeh, D., Tol, A., Yaseri, M., \& Rohban, A. (2018). Depression, Anxiety , and Perceived Social Support among Adults with Beta- Thalassemia Major : Cross-Sectional Study, 101-107.

OMS. (2018). Obesidad y Sobrepeso. Recuperado el 15 de diciembre de 2018, a partir de https://www.who.int/es/news-room/fact-sheets/detail/obesity-and-overweight

Osborn, C. Y., Ph, D., H, M. P., Egede, L. E., \& S, M. (2012). The relationship between depressive symptoms and medication nonadherence in type 2 diabetes : the role of social support , General Hospital Psychiatry, 34(3), 249-253. https://doi.org/10.1016/j.genhosppsych.2012.01.015

Parro Moreno A, Santiago Pérez IM, Abraira V, Aréjula Torres JL, Gandarillas-Grande A, Díaz Holgado A, Morales Asencio JM, S. P. (2016). Control de la diabetes mellitus en población adulta según las características del personal de enfermería de atención primaria de la comunidad de Madrid: Análisis multinivel. Revista Española de Salud Pública, 90(4).

Primaria, D. G. de A. (2016). Informe Estado de Salud de la Población de la Comunidad de Madrid: objetivo 5 Envejecer en buena salud. Recuperado el 30 de noviembre de 2018, a partir de http://www.comunidad.madrid/sites/default/files/doc/sanidad/epid/ies2016_objetivo5.pdf 
Ramkisson, S. (2017). Social support and coping in adults with type 2 diabetes. African Journal of Primary Health Care and Family Medicine, 9(1), 1-8.

Risk, N. C. D., \& Collaboration, F. (2016). Worldwide trends in diabetes since 1980 : a pooled analysis of 751 population-based studies with 4.4 million participants. The Lancet, 387(10027), 1513-1530. https://doi.org/10.1016/S0140-6736(16)00618-8

Rotella F, M. E. (2013). Diabetes mellitus as a risk factor for depression. Diabetes research and clinical practice, 2(98), 98-104.

Smalls, B. L., A, M. H. S., Walker, R. J., S, M., Hernandez-tejada, M. A., S, M., ... S, M. (2012). Associations between coping, diabetes knowledge, medication adherence and self-care behaviors in adults with type 2 diabetes General Hospital Psychiatry, 34(4), 385-389. https://doi.org/10.1016/j.genhosppsych.2012.03.018

Soler-González, J., Marsal, J. R., Serna, C., Real, J., Cruz, I., \& López, A. (2013). La población inmigrante controla peor su diabetes que la población autóctona. Gaceta Sanitaria, 27(1), 19-25. https://doi.org/10.1016/j.gaceta.2011.12.015

Tovar, E., Rayens, M. K., Gokun, Y., \& Clark, M. (2013). Mediators of adherence among adults with comorbid diabetes and depression: The role of self-efficacy and social support. Journal of Health Psychology, 20(11), 1405-1415. https://doi.org/10.1177/1359105313512514

Val Jiménez A, Amorós Ballestero G, Martínez Visa P, Fernández Ferré ML, L. S. M. (1992). Descriptive study of patient compliance in pharmacologic antihypertensive treatment and validation of the Morisky and Green test. Atención Primariarimaria, 10(5), 767-770.

WHO. (2016). World Health Organization. Global status report on noncommunicable disease, Diabetes. Recuperado a partir de https://www.who.int/diabetes/country-profiles/mex_es.pdf?ua=1

WHO. (2018). World Health Organization. Global Report on Diabetes. Recuperado a partir de https://www.who.int/diabetes/global-report/en/

Williams, A., Sethi, B., Duggleby, W., Ploeg, J., Markle-reid, M., \& Peacock, S. (2016). A Canadian qualitative study exploring the diversity of the experience of family caregivers of older adults with multiple chronic conditions using a social location perspective. International Journal for Equity in Health, 1-16. https://doi.org/10.1186/s12939-016-0328-6

Wonca. (1999). Clasificación Internacional de Atención Primaria CIAP-2 (depresión y ansiedad). Recuperado el 20 de agosto de 2006, a partir de https://www.iqb.es/patologia/ciap/ciap_p.htm

WONCA. (1999). Clasificación Internacional de Atención Primaria CIAP-2 Diabetes. Recuperado el 28 de noviembre de 2018, a partir de https://www.iqb.es/patologia/ciap/ciap_t.htm 